\title{
Proper Orthogonal Decomposition of the Unsteady Pressure Field in the Three-Dimensional Wall Jet
}

\author{
Bonnie Sim \\ Department of Mechanical Engineering \\ University of New Brunswick \\ Fredericton, Canada \\ bonnie.sim@unb.ca
}

\author{
Joseph W. Hall \\ Department of Mechanical Engineering \\ University of New Brunswick \\ Fredericton, Canada \\ jwhall@unb.ca
}

\begin{abstract}
The streamwise variation in the unsteady wall pressure of a turbulent three-dimensional wall jet was examined using Proper Orthogonal Decompostion. Based on the exit velocity and the nozzle diameter, the Reynolds number of the wall jet was 140,000 . The fluctuating wall pressure was measured using a two-dimensional lateral-streamwise array of 89 condenser microphones from $x / D=5$ to 15 . The instantaneous pressure fluctuations showed strong lateral antisymmetry, which appeared to be linked to the lateral development of the flow. Instantaneous intermittent events convected downstream through the entire measurement region but were observed to change speed, slow down, surge forward, and convect laterally. The first two POD modes were antisymmetric and the next few higher modes appeared to be associated with lateral meandering of the jet. Loworder reconstructions of fluctuating wall pressure displayed the intermittency and meandering observed in the unsteady pressure
\end{abstract} field.

Index Terms-Three-dimensional wall jets, unsteady wall pressure

\section{INTRODUCTION}

Three-dimensional wall jets are flows that exit from finitewidth openings and develop tangentially to a surface. Wall jets share characteristics with free jets and boundary layer flows [1]-[3], and are widely used in industry, primarily for heating and cooling. These jets are unique because they exhibit a comparatively large lateral growth rate in the farfield, approximately five to eight times larger than the wallnormal growth rate [3], [4]. While the mechanisms that cause this disparity in growth rates are not fully explained, the large lateral growth has been shown to be caused by strong mean turbulence generated secondary flows in the jet [2], [4], [5]. These strong secondary flows are oriented to draw the mean flow downward and eject it laterally outward.

Many researchers have recognized that secondary flow is linked to the passage of coherent structures in the flow. Coherent structure models in the wall jet have been developed in several studies, including those conducted by Matsuda et al [6], Ewing and Pollard [7], [8], Sun and Ewing [9]-[11], Hall and Ewing [12]-[16], and Namgyal and Hall [5], [17][19]. In general, most research indicates that there are large

The authors are grateful for funding by the Natural Sciences and Engineering Research Council of Canada (NSERC), the New Brunswick Innovation Foundation (NBIF), and the International Society of Automation Process Measurement and Control Division (ISA PMCD). horseshoe-like vortices, which are responsible for a downward and lateral ejection of the flow [16], [18]. The present body of research suggests that these structures are likely asymmetric with respect to the jet centreline [15], [18], [19]. A recent coherent structure model formulated by Namgyal and Hall [18], [19] proposes two dominant types of structures; structures in the outer shear layer that develop from instabilities in the vortex rings shed from the nozzle and are responsible for entraining ambient air, and near-wall structures that persist into the intermediate- and far-fields of the wall jet. The asymmetric passage of these structures causes large intermittent lateral ejections of flow, and Namgyal and Hall [18], [19] theorized that the interaction between the outer and inner structures strengthens these lateral ejections.

Although these previous studies have been essential in developing the coherent structure model for three-dimensional wall jets, they have limitations. The majority of the analysis and development of these models was conducted using timeaveraged information; many of the earlier studies [6]-[9], [11] used single-point or two-point velocity measurements in conjunction with averaging to develop the models and make inferences about structure formation. Therefore, the instantaneous dynamics of the large-scale structures could not be incorporated into the models. Additionally, these previous studies [5], [7]-[19] utilized velocity measurements at discrete streamwise positions and were unable to account for the temporal-streamwise variation of the structures in the wall jet. Of the previous models, only Hall and Ewing's [13]-[16] incorporated fluctuating wall pressure measured at singular streamwise positions to deduce structural dynamics dynamics.

In a recent study by Sim and Hall [20], some of these limitations were addressed through measurements of the fluctuating wall pressure over a two-dimensional array in the wall jet. Since the microphone array encompassed a large twodimensional area, the instantaneous pressure measurements could be used to assess the streamwise variation in the wall jet and investigate the continuous evolution of the coherent structures. On average, the spectral analysis suggested that the passage of the structures occurred at a fairly constant convection speed. However, an examination of the instantaneous unsteady fluctuating wall pressure suggested that the convection speed was not constant and that structures did 


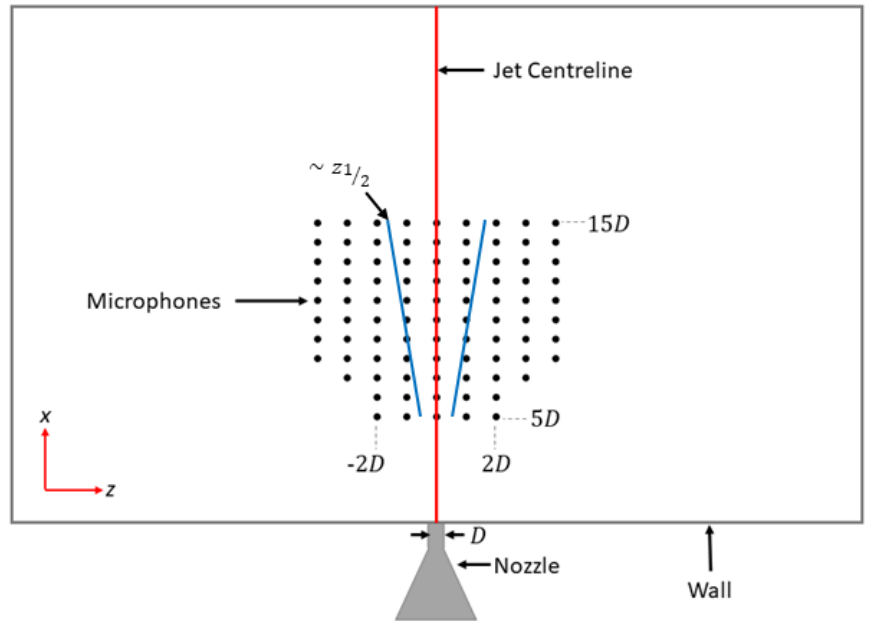

Fig. 1: Microphone array layout.

not always convect directly downstream [20]. Furthermore, it was observed that, instantaneously, there were angled regions of organization that appeared to be associated with lateral movement of the flow. The current study aims to investigate in more detail the variation of the unsteady pressure field of the turbulent wall jet, and in particular the angled nature of the structures, using Proper Orthogonal Decomposition to better understand the angled structures and convection speed.

\section{Experimental Methodology}

The experimental set-up is the same as was used in the previous study by Sim and Hall [20] and utilized a threedimensional wall jet exiting from a contoured nozzle. The details for the experimental set up are outlined in [20]. Based on the exit diameter, the Reynolds number was $R e_{D} \approx$ 140,000 , which corresponded to a nominal exit velocity of $U_{\text {exit }} \approx 54.4 \mathrm{~m} / \mathrm{s}$. To investigate the streamwise evolution of the coherent structures in the three-dimensional wall jet, the fluctuating wall pressure was simultaneously measured by 89 CUI electret condenser microphones in a two-dimensional array, as shown in Fig. 1. The microphones were positioned at one-diameter intervals from $5 \leq x / D \leq 15$ downstream from the nozzle exit. The lateral positions of the microphones ranged from $-4 \leq z / D \leq 4$ at one-diameter intervals, which spanned at least twice the lateral half-width of the jet (shown on Fig. 1). The microphones had a flat frequency response from $40 \mathrm{~Hz}-1 \mathrm{kHz}$ and were simultaneously sampled at a rate of $10 \mathrm{kHz}$.

Fluctuating wall pressure statistics were calculated using $1.5 \times 10^{7}$ data points yielding an uncertainty in the standard deviation of $<0.05 \%$ at a $95 \%$ confidence interval. Spectra and correlations were calculated by averaging the results of 1500 one-second blocks of fluctuating wall pressure comprising $1 \times 10^{4}$ data points. The uncertainty in the magnitude of the pressure spectra was $<2.6 \%$ at a $95 \%$ confidence interval.

\section{Proper Orthogonal Decomposition}

The technique of Proper Orthogonal Decomposition (POD) can be used to represent the fluctuating wall pressure using a set of orthogonal basis functions obtained by maximizing the pressure fluctuations on these basis functions, which yields the optimal representation for the flow. This technique has been used by many [16], [21]-[24] to identify coherent structures in turbulent flows. The functions are the solutions to the integral eigevenvalue problem given by [25]:

$$
\int_{-\infty}^{\infty} \phi\left[(x, z),\left(x^{\prime}, z^{\prime}\right)\right] \psi\left(x^{\prime}, z^{\prime}\right) d x^{\prime} d z^{\prime}=\lambda^{(n)} \psi(x, z),
$$

where $\psi(x, z)$ are the eigenvectors, $\lambda^{(n)}$ are the eigenvalues of the $n$ modes, and $\phi\left[(x, z),\left(x^{\prime}, z^{\prime}\right)\right]$ is the cross-correlation tensor with zero time lag $(\tau=0)$. The cross-correlation tensor is given by:

$$
\phi\left[(x, z),\left(x^{\prime}, z^{\prime}\right)\right]=\left\langle p^{\prime}(x, z) p^{\prime}\left(x^{\prime}, z^{\prime}\right)\right\rangle,
$$

where $p^{\prime}$ is the measured fluctuating wall pressure at the indicated position. The relative pressure energy recovered using each POD mode is given by:

$$
\zeta_{R}^{(n)}=\frac{\lambda^{(n)}}{\sum_{i=1}^{m} \lambda^{(m)}} .
$$

The coefficients of each POD mode are given by:

$$
a^{(n)}=\int p^{\prime}(x, z) \psi^{(n)}(x, z) d x d z .
$$

The first $N$ POD modes and coefficients can be used to reconstruct the spatially filtered pressure field using

$$
p_{\text {rec }, n}^{\prime}(x, z)=\sum_{n=1}^{N} a_{n} \psi(x, z) .
$$

A low-dimensional reconstruction of the unsteady pressure field can be generated by using the first $N$ modes for the reconstruction, per:

$$
\begin{aligned}
p_{\mathrm{rec}, n=1 \ldots 3}^{\prime}(x, z) & =\sum_{n=1}^{N=3} a_{n} \psi(x, z) \\
& =a_{1} \psi(x, z)+a_{2} \psi(x, z)+a_{3} \psi(x, z) .
\end{aligned}
$$

\section{RESUlts AND ANALYsis}

\section{A. Instantaneous Fluctuating Wall Pressure}

To gain a feel for the pressure fluctuations in the wall jet, an unfiltered instant is shown in Fiure 2. The unsteady pressure field here exhibited asymmetric and antisymmetric pressure fluctuations about the jet centreline, as shown in Fig. 2. The lateral asymmetry has been previously observed by [13]-[16] and more recently by Sim and Hall [20]. The lateral asymmetry appeared over the region of the wall jet examined and starts at or before $x / D=5$. The fluctuations often appear to be connected across the centreline in an angled fashion. When these angled regions occur in succession, as shown in Fig. 3, the strong laterally antisymmetric fluctuations cause 


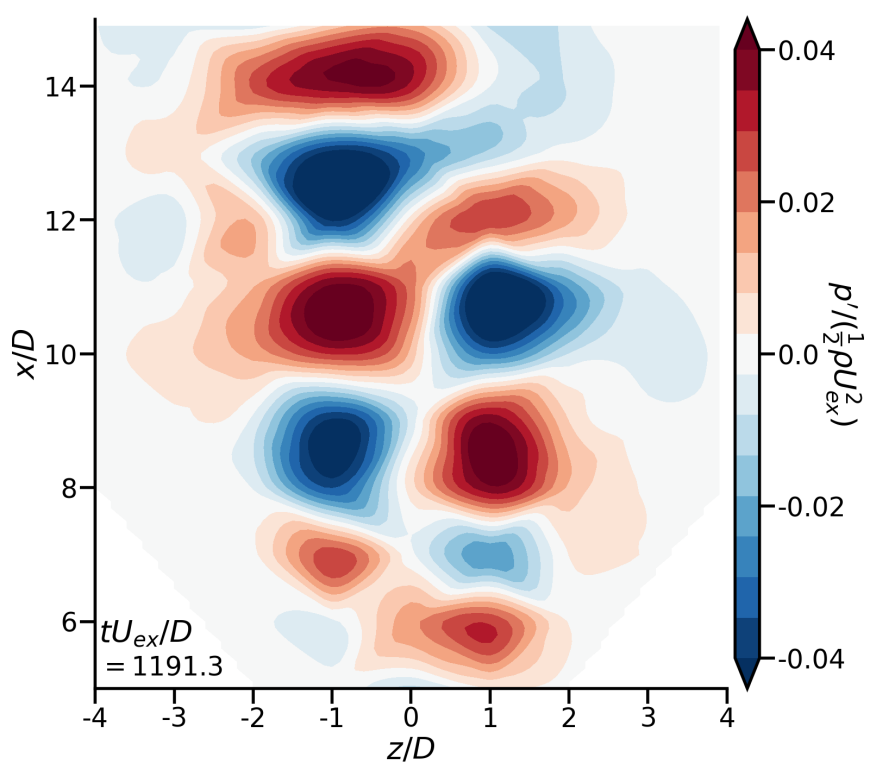

Fig. 2: Example of asymmetry in the unsteady pressure field.

the flow to be driven laterally outward to the right (Fig. 3a) or the left (Fig. 3b), in a process identified as meandering.

\section{B. Proper Orthogonal Decomposition}

The streamwise variation and organization of the unsteady pressure field was further examined by applying POD to the fluctuating wall pressure. The relative and cumulative amount of energy recovered by each POD mode is shown in Fig. 4. The POD converges relatively quickly, with $50 \%$ of the pressure energy recovered in the first 12 modes, where there are 89 modes in total. The lower modes appear to contain very similar quantities of energy, with Mode 1 at $5.72 \%$ and Mode 12 at $3.16 \%$.

The mode shapes for the first twelve POD modes are shown in Fig. 5. Modes 1 and 2 are primarily antisymmetric about the jet centreline, which is consistent with previous work [13]-[16], [20], and appear to be the inverse of each other. They have relatively similar energy levels, $5.72 \%$ compared to $5.43 \%$, and appear to have peaks that align with the lateral half-width of the jet, as indicated by the dashed lines on the mode shapes.

Mode 3 is loosely symmetric about the centreline; it may be representative of the connection between regions of organized pressure across the centreline. However, in the near field at $x / D \leq 10$, Mode 3 and Mode 4 appear to be similar in that there are larger regions of organized pressure fluctuations that are tilted outward. Mode 3 appears to have this tilted pressure angled out to the left (similar to the leftward meander shown in Fig. 3b), while Mode 4 displays tilted pressure that is angled to the right (similar to the rightward meander shown in Fig. 3a). Downstream of $x / D=10$, the leftward tilt in Mode 3 appears to decrease, while the rightward meander in Mode 4 is accentuated.
Higher number modes (Modes 5, 6, 8, 9, 10, and 12) also appear to contribute to or to also be representative of lateral meandering in the wall jet. Modes that appear to be associated with the meandering exhibit stronger organization on one side of the wall jet, and usually appear to strengthen with downstream position. There are other modes (Modes 7 and 11) that are localized to the centreline and shows regions of organization of different size. These modes could be associated with changes in structure size or convection speed at the centreline, and could be linked to structures of different frequencies, which were previously suggested by Hall and Ewing [13], [16].

In order to investigate how POD recovers the lateral meandering in the the wall jet, the two-dimensional unsteady pressure field was reconstructed using specific modes. The modes used for the reconstructions were selected because the appeared to be connected to either leftward or rightward meandering in the wall jet. These modes displayed stronger fluctuations on one side of the centreline and/or angling in the regions of organized pressure fluctuations consistent with the lateral angling shown in the instances in Fig. 3. There were modes selected for reconstructing to show leftward and rightward meandering in the unsteady pressure field; these reconstructions in addition to the corresponding unfiltered pressure instants are shown in Fig. 6 (Figures 6a, 6d, 6g, and $6 j)$.

The sequences shown in Fig. 6 show instants leading up to and after the leftward meandering field shown in Fig. 3b. To illustrate the effect of the selective mode choice in the reconstructions, the reconstructed pressure field is shown in Fig. 6 using two sets of modes: those that appear to correspond to leftward meandering and those that appear to correspond to rightward meandering. Leftward meandering appears to be connected to the contributions from Modes 2, 3, 5, 6, and 9, and is shown in the middle sequence in Fig. 6 (Figures $6 \mathrm{~b}, 6 \mathrm{e}, 6 \mathrm{~h}$, and $6 \mathrm{k}$ ). There are other higher modes that would also contribute to leftward meandering, especially at increased downstream distances, but these lower modes show the angled growth of the wall jet in the near field and capture $22.43 \%$ of the energy in the flow.

Not as apparent in this sequence, the pressure has also been reconstructed with modes associate with rightward meandering, shown in right column of Figure 6 (Figures 6c, 6f, 6f, and 61 ). Modes 1, 4, 8, 10, and 12 contain approximately $20.73 \%$ of the energy and appear to be linked to rightward meandering. Since the unfiltered sequence shown in Fig. 6 is associated with leftward meandering of the wall jet, it is expected that the reconstruction with these modes would contain lower comparable energy and organization. Although not shown here for the sake of brevity, reconstructions with these modes do indicate that these are tied to rightward meandering. This sequence and reconstructions with these sets of modes show pressure fields that are consistently laterally angled or meandering primarily in one direction, either leftward or rightward. Therefore, it suggests that there are specific modes or sequences of modes that are representative of meandering in the wall jet; targeting 


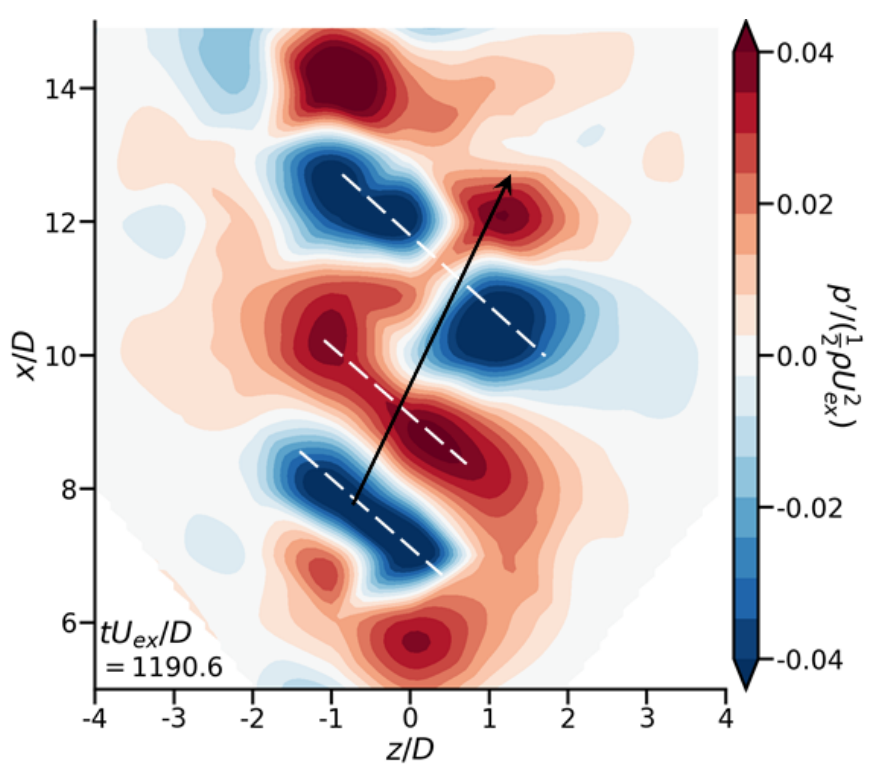

(a) Rightward meandering

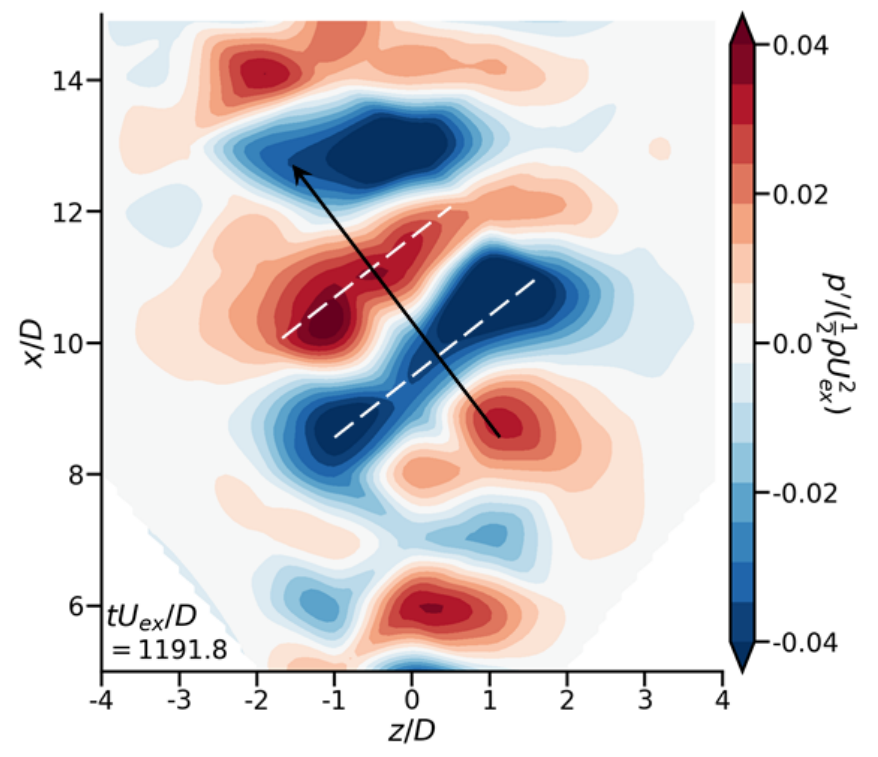

(b) Leftward meandering

Fig. 3: Meandering in the three-dimensional wall jet.

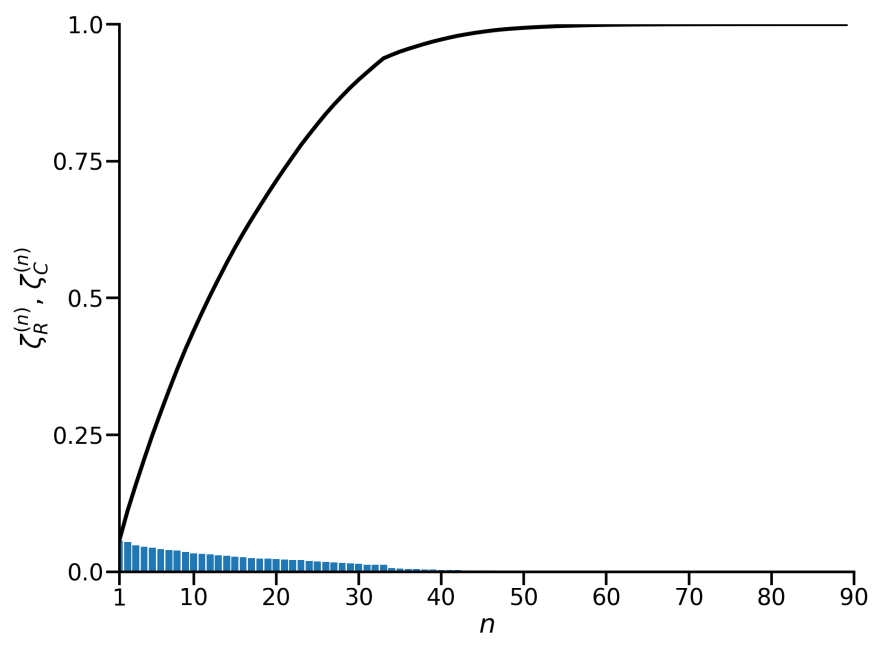

Fig. 4: Relative and cumulative pressure energy recovered using POD.

these modes could amplify lateral jet development.

\section{CONCLUSIONS}

The fluctuating wall pressure was experimentally measured in the three-dimensional wall jet. Instantaneously, there was strong lateral asymmetry and antisymmetry observed in the unsteady pressure field. The strong antisymmetry is linked to angled packets of organized pressure and strong lateral meandering that contribute to the lateral development of the wall jet. The organization of the unsteady pressure field of the three-dimensional wall jet was further analyzed using POD. Low-order reconstructions allowed lateral meandering in the wall jet to be examined further. Reconstructing the unsteady pressure field with select modes allowed meandering on either side of the wall jet to be isolated; there were specific modes associated with leftward meandering and other modes associated with rightward meandering.

The future aims of this study are to apply spectral POD to the fluctuating wall pressure to allow further insight into the link between lateral meandering in the wall jet and frequencies. Since it appears that specific modes are connected to lateral jet development, determining the characteristic frequencies of these modes would potentially allow control of lateral growth in the jet. Identification of key frequencies in the flow would provide a basis for targeted active flow control, similar to the work of [26].

\section{REFERENCES}

[1] P. Bakke, "An experimental investigation of a wall jet," J. Fluid Mech., vol. 2, no. 5, pp. 467-472, 1957.

[2] B. E. Launder and W. Rodi, "The turbulent wall jet measurements and modeling," Ann. Rev. Fluid Mech., vol. 15, no. 1, pp. 429-459, 1983.

[3] B. E. Launder and W. Rodi, "The turbulent wall jet," Prog. Aerosp. Sci., vol. 19 , pp. $81-128,1979$.

[4] T. J. Craft and B. E. Launder, "On the spreading mechanism of the three-dimensional turbulent wall jet," J. Fluid Mech., vol. 435, no. 1, pp. 305-326, 2001.

[5] L. Namgyal and J. W. Hall, "Reynolds stress distribution and turbulence generated secondary flow in the turbulent three-dimensional wall jet," $J$. Fluid Mech., vol. 800, pp. 613-644, 2016.

[6] H. Matsuda, S. Iida, and M. Hayakawa, "Coherent structures in a threedimensional wall jet," J. Fluids Eng., vol. 112, no. 4, p. 462, 1990.

[7] D. Ewing and A. Pollard, "Evolution of the large scale motions in a three-dimensional wall jet," in $28^{\text {th }}$ Fluid Dyn. Co-located Conf., American Institute of Aeronautics and Astronautics, June 1997.

[8] D. Ewing, A. Benaissa, A. Pollard, J. Citriniti, H. Abrahamsson, and L. Lofdahl, "Contribution of large structures to the anisotropic spread rate in a wall jet issuing from a round nozzle," in Tenth Int. Symp. Transp. Phenom. Therm. Sci. Process Eng., 1997.

[9] H. Sun, Development of Three-Dimensional Turbulent Wall Jets. PhD thesis, McMaster University, 2002.

[10] H. Sun and D. Ewing, "The development of three-dimensional wall jet," in Proc. $48^{\text {th }}$ Ann. Conf. Canadian Aeronautics and Space Institute, (Toronto, Canada), 2002. 


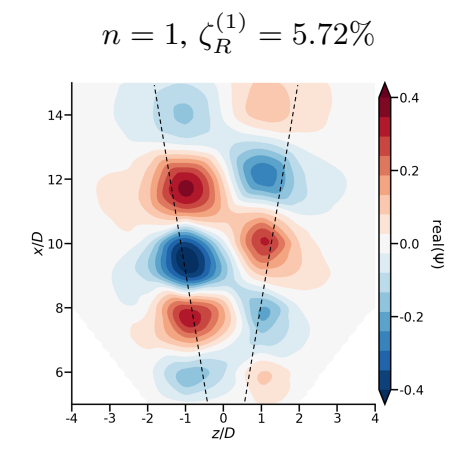

$$
n=2, \zeta_{R}^{(2)}=5.43 \%
$$

$n=3, \zeta_{R}^{(3)}=4.80 \%$

$n=4, \zeta_{R}^{(4)}=4.59 \%$
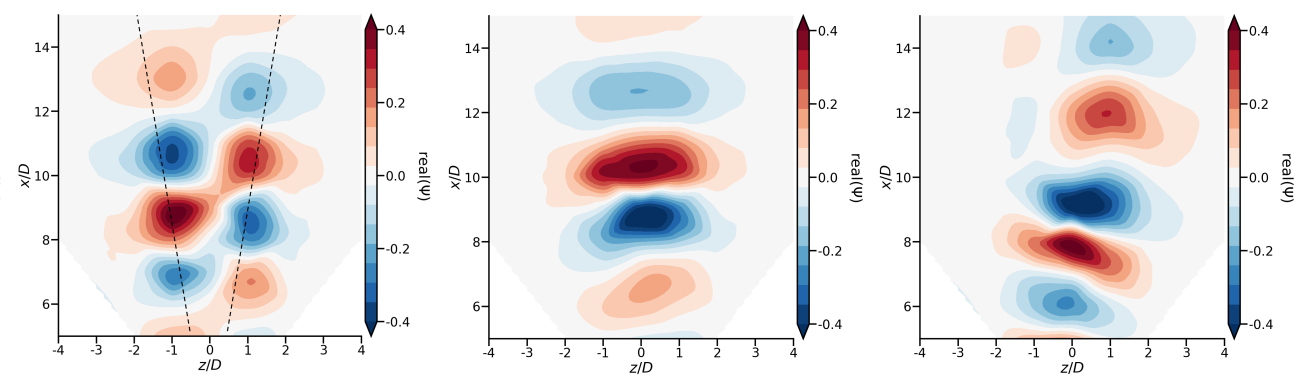

$$
n=5, \zeta_{R}^{(5)}=4.41 \%
$$

$n=6, \zeta_{R}^{(6)}=4.13 \%$

$$
n=7, \zeta_{R}^{(7)}=4.01 \%
$$

$n=8, \zeta_{R}^{(8)}=3.87 \%$
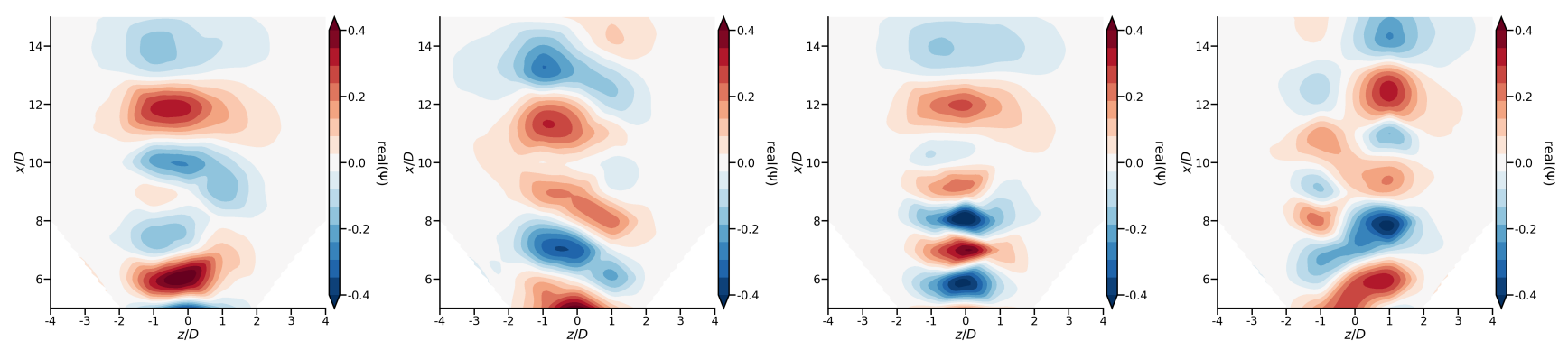

$$
n=9, \zeta_{R}^{(9)}=3.66 \%
$$

$n=10, \zeta_{R}^{(10)}=3.39 \%$

$$
n=11, \zeta_{R}^{(11)}=3.27 \%
$$

$$
n=12, \zeta_{R}^{(12)}=3.16 \%
$$
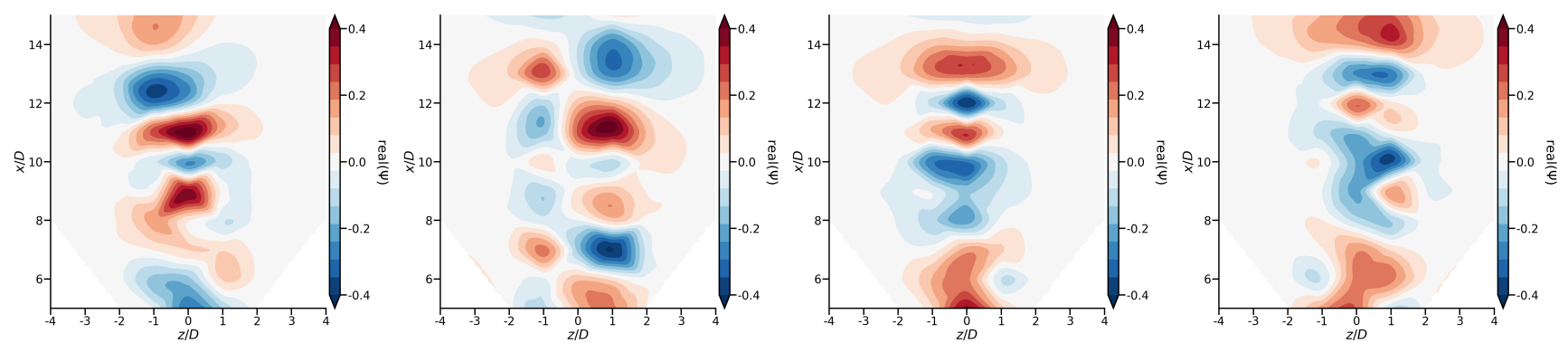

Fig. 5: POD modes

[11] H. Sun and D. Ewing, "Effect of initial and boundary conditions on development of three-dimensional wall jets," AIAA paper 2002-0777, McMaster University, 2002.

[12] J. W. Hall and D. Ewing, "On the dynamics of the large-scale structures in round impinging jets," J. Fluid Mech., vol. 555, pp. 439-438, 2006.

[13] J. W. Hall and D. Ewing, "A combined spatial and temporal decomposition of the coherent structures in the three-dimensional wall jet," in 44th AIAA Aerospace Sciences Meeting and Exhibit, (Reno, NV USA), pp. 1-18, January 2006.

[14] J. W. Hall and D. Ewing, "Three-dimensional turbulent wall jets issuing from moderate-aspect-ratio rectangular channels," AIAA J., vol. 45, no. 6 , pp. 1177-1186, 2007.

[15] J. W. Hall and D. Ewing, "The asymmetry of the large-scale structures in turbulent three-dimensional wall jets exiting long rectangular channels," J. Fluids Eng., vol. 129, no. 7, pp. 929-941, 2007.

[16] J. W. Hall and D. Ewing, "Spectral linear stochastic estimation of the turbulent velocity in a square three-dimensional wall jet," J. Fluids Eng., vol. 132, no. 5, pp. 1-9, 2010.

[17] L. Namgyal, Three-Component Particle Image Velocimetry Measurements in a Turbulent Three-Dimensional Wall Jet. PhD thesis, Unversity of New Brunswick, Fredericton, New Brunswick, Canada, March 2012.

[18] L. Namgyal and J. W. Hall, "Coherent streamwise vortex structures in the near-field of a the three-dimensional wall jet," J. Fluids Eng., vol. 135 , no. 6 , pp. 1-7, 2013.
[19] L. Namgyal and J. W. Hall, "Coherent streamwise vortex structures of the turbulent three-dimensional wall jet," Submitt. to J. Fluid Mech., 2015.

[20] B. Sim and J. Hall, "Streamwise variation of the unsteady pressure field in the three-dimensional wall jet," in AIAA Scitech 2020 Forum, (Orlando, FL, USA), January 2020.

[21] M. N. Glauser, S. J. Leib, and W. K. George, Coherent Structures in the Axisymmetric Jet Mixing Layer. Berlin: Springer, 1987.

[22] J. Delville, L. Ukeiley, L. Cordier, J. P. Bonnet, and M. Glauser, "Examination of large-scale structures in a turbulent plane mixing layer. part 1. proper orthogonal decomposition," J. Fluid Mech., vol. 391, pp. $91-122,1999$.

[23] J. A. Taylor and M. N. Glauser, "Towards practical flow sensing and control via POD and LSE based low-dimensional tools," J. Fluids Eng., vol. 126, no. 3, pp. 337-345, 2004.

[24] C. E. Tinney, L. S. Ukeiley, and M. N. Glauser, "Low-dimensional characteristics of a transonic jet. Part 2. Estimate and far-field prediction," J. Fluid Mech., vol. 615, no. 1, pp. 53-92, 2008.

[25] G. Berkooz, P. Holmes, and J. L. Lumley, "The Proper Orthogonal Decomposition in the Analysis of Turbulent Flows," Annual Review of Fluid Mechanics, vol. 25, pp. 539-575, jan 1993.

[26] N. A. Worth, D. Mistry, T. Berk, and J. R. Dawson, "Vortex dynamics of a jet at the pressure node in a standing wave," J. Fluid Mech., vol. 882, pp. A22-1 - A22-27, 2020. 
(a) Unfiltered $p^{\prime}$

(b) $\Sigma p_{n}^{\prime}, n=2,3,5,6,9$

(c) $\Sigma p_{n}^{\prime}, n=1,4,8,10,12$

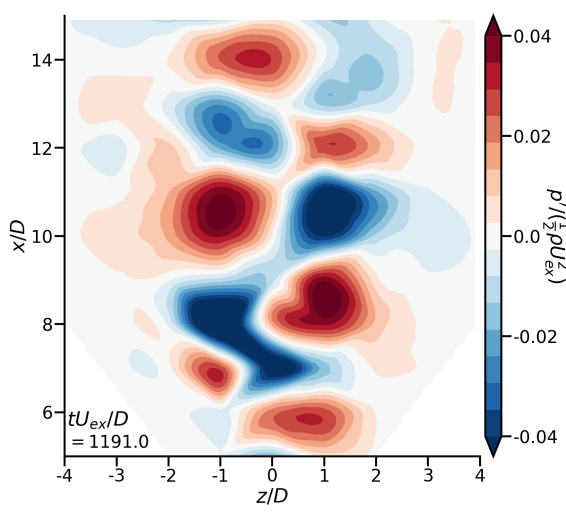

(d) Unfiltered $p^{\prime}$

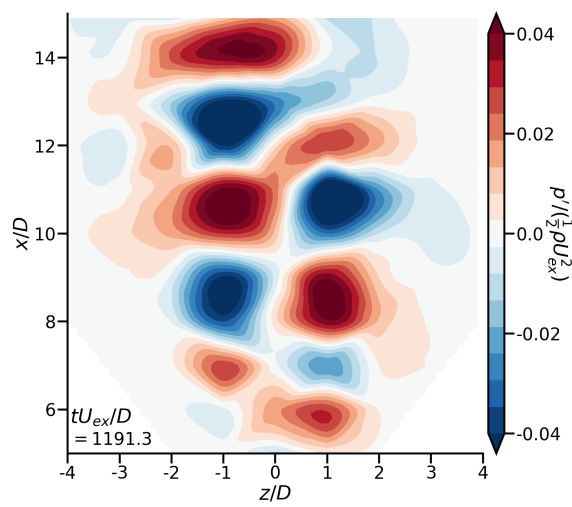

(g) Unfiltered $p^{\prime}$

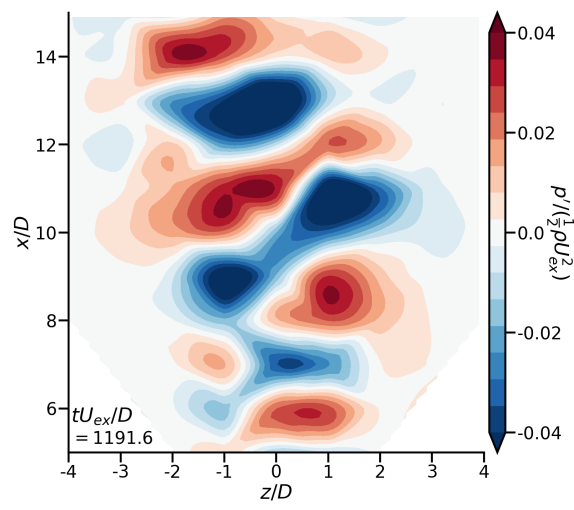

(j) Unfiltered $p^{\prime}$

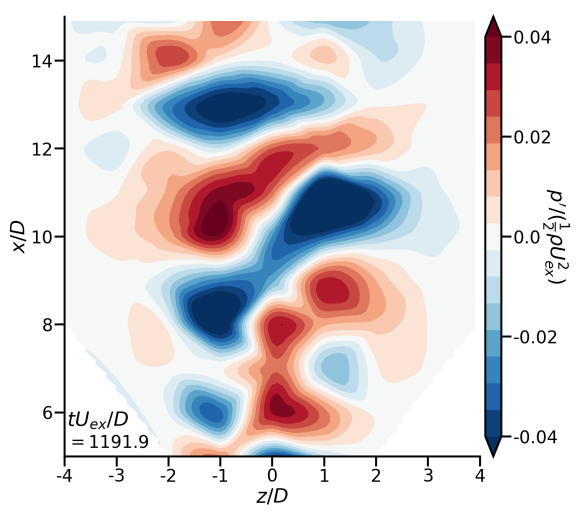

(e) $\Sigma p_{n}^{\prime}, n=2,3,5,6,9$
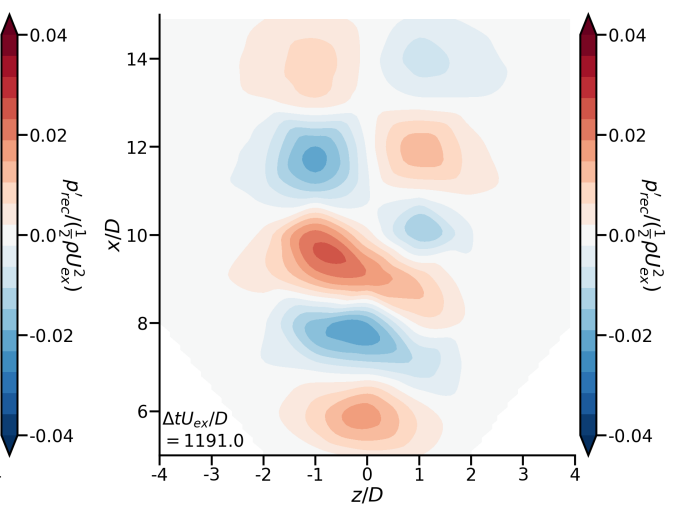

(f) $\Sigma p_{n}^{\prime}, n=1,4,8,10,12$

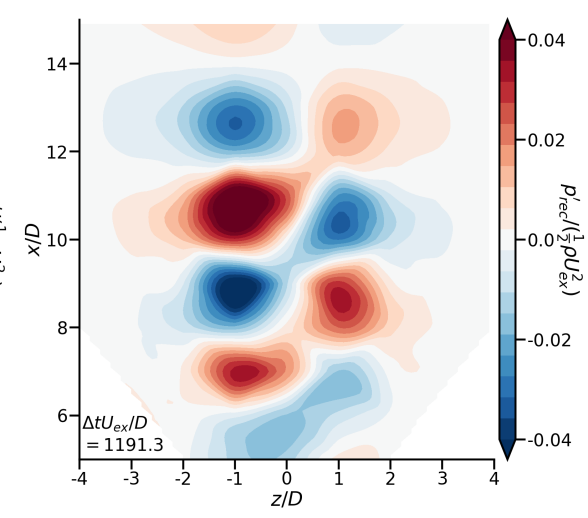

(h) $\Sigma p_{n}^{\prime}, n=2,3,5,6,9$

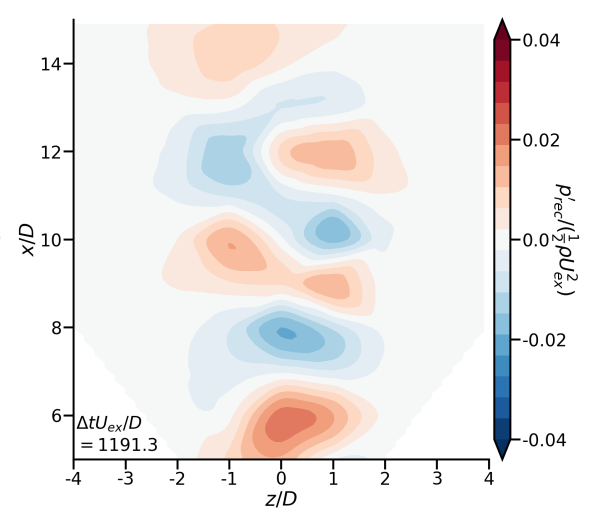

(i) $\Sigma p_{n}^{\prime}, n=1,4,8,10,12$

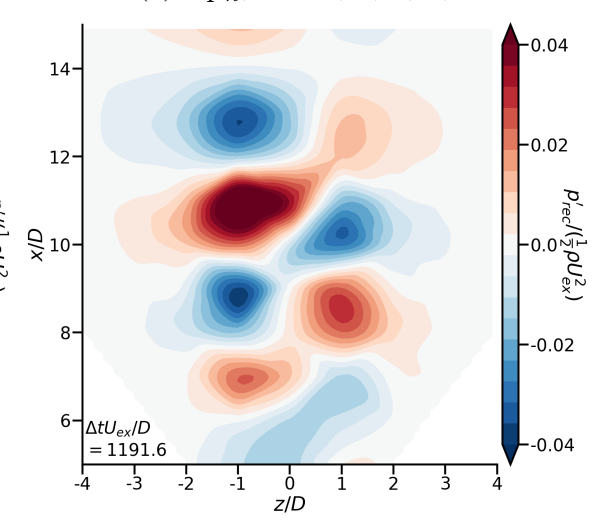

(k) $\Sigma p_{n}^{\prime}, n=2,3,5,6,9$

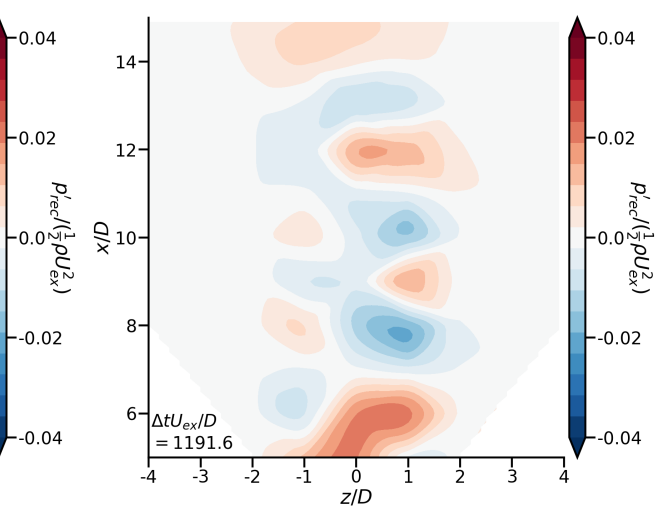

(1) $\Sigma p_{n}^{\prime}, n=1,4,8,10,12$
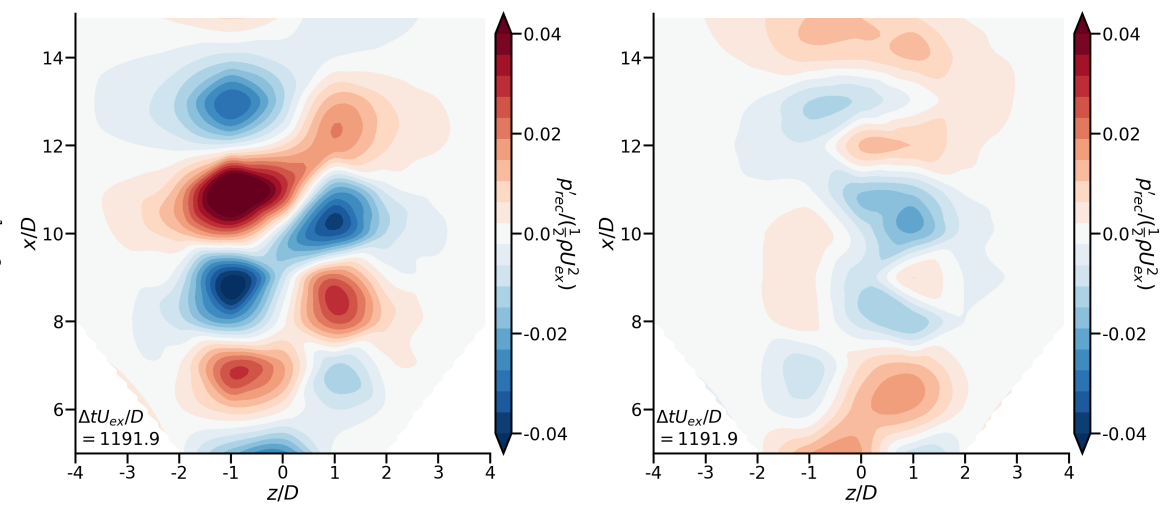

Fig. 6: Instances of reconstructed pressure compared to the experimentally measured fluctuating wall pressure. 